\title{
A Dynamic Algorithm for Facilitated Charging of Plug-In Electric Vehicles
}

\author{
Nicole Taheri* $\quad$ Robert Entriken ${ }^{\dagger} \quad$ Yinyu Ye Ye $^{\ddagger}$
}

November 9, 2018

\begin{abstract}
Plug-in Electric Vehicles (PEVs) are a rapidly developing technology that can reduce greenhouse gas emissions and change the way vehicles obtain power. PEV charging stations will most likely be available at home and at work, and occasionally be publicly available, offering flexible charging options. Ideally, each vehicle will charge during periods when electricity prices are relatively low, to minimize the cost to the consumer and maximize societal benefits. A Demand Response (DR) service for a fleet of PEVs could yield such charging schedules by regulating consumer electricity use during certain time periods, in order to meet an obligation to the market.

We construct an automated DR mechanism for a fleet of PEVs that facilitates vehicle charging to ensure the demands of the vehicles and the market are met. Our dynamic algorithm depends only on the knowledge of a few hundred driving behaviors from a previous similar day, and uses a simple adjusted pricing scheme to instantly assign feasible and satisfactory charging schedules to thousands of vehicles in a fleet as they plug-in. The charging schedules generated using our adjusted pricing scheme can ensure that a new demand peak is not created and can reduce the consumer cost by over $30 \%$ when compared to standard charging, which may also increase peak demand by $3.5 \%$. In this paper, we present our formulation, algorithm and results.
\end{abstract}

\section{Introduction}

A Plug-in Electric Vehicle (PEV) is any vehicle that uses electricity from the grid to displace liquid fuel. These vehicles will be able to plug-in to the grid and control which times during the connection period the vehicle battery will actually charge. This capability will allow consumers to charge their vehicles during off-peak hours, usually also resulting in a reduced cost to the consumer. For example, suppose a vehicle is plugged in from $6 \mathrm{pm}$ until 7am, giving an 11-hour window in which the vehicle can charge. If the battery can obtain sufficient charge in one hour, this charge can be scheduled to occur during an ideal hour between $6 \mathrm{pm}$ and $7 \mathrm{am}$, which takes into account the electricity price, electricity load, and individual vehicle battery state of charge and driving schedule. This will reduce consumer cost of charging and help cultivate a balance between the electricity supply and the demand.

\footnotetext{
*Institute for Computational and Mathematical Engineering, Stanford University, Stanford, CA. Email: ntaheri@stanford.edu.

${ }^{\dagger}$ Electric Power Research Institute, Palo Alto, CA. Email: rentrike@epri.com.

${ }^{\ddagger}$ Department of Management Science and Engineering, Stanford University, Stanford, CA. Email: yinyu-ye@stanford.edu.
} 
In order for PEV charging to be advantageous for both the PEV owners and the utility, a mechanism is needed that receives information from both the vehicles and the system dispatcher to facilitate vehicle charging. In this paper, we design an algorithm for such a mechanism that will be used by an aggregator.

Definition 1 An aggregator manages the communication and electricity distribution between a group of electricity consumers and an electric utility.

Currently, aggregators regulate consumer electricity use by rewarding decreases in electricity demand during certain time periods in order to meet a scheduling obligation to the market or system dispatcher. These Demand Response (DR) resources are provided by either third-parties (such as EnerNOC and ZigBee Smart Energy) or the utilities themselves (for example, both PG\&E and Duke Energy have DR programs, among many others). Existing programs focus on the Commercial and Industrial Sector, with a small number of large customers who typically use hundreds of kilowatt hours $(\mathrm{kWh})$ per day [23]. These programs are not automated, requiring human intervention to individually tailor the DR resource for each customer and monitor the electricity use of each customer alongside the market supply and prices.

By contrast, some estimates say there may be 100 million PEVs on the road in the United States by 2030 [9] and each PEV battery will generally require a few kWh each day. Thus, an automated DR program would be more convenient and scalable to large fleets of vehicles than the alternative of individually tailoring human-operated DR for each consumer. An automated DR service can manage the transactions between a fleet of PEVs (say, around 10,000) and their utility, facilitating the charging schedules of the fleet to meet the transportation demands of the vehicle owners and meet a scheduling obligation.

In this paper, we describe an automated mechanism that performs Demand Response services with a fleet of Plug-In Electric Vehicles. Our algorithm constructs adjusted 'prices' for each hour that take into account a scheduling obligation, transportation demands of the fleet, vehicle driving schedules, and current electricity pricing. Vehicle charging schedules will be based on these adjusted prices and determined instantly as vehicles plug-in, without knowing the energy needs of subsequent vehicles connecting. The resulting charging schedules will meet the energy demands of all vehicles in the fleet while avoiding increased load during certain time periods, in order to meet the scheduling obligations. For example, our algorithm can help ensure that a new peak is not created while meeting vehicle charging needs, or that the added amount of electrical load at any time is below a given threshold, which is a key feature to maintaining the stability of the power grid.

Our mechanism can be implemented as a device attached to each vehicle in a fleet; these devices can communicate with each other and regulate vehicle charging. Installing the device on a vehicle will result in two main benefits for the consumer: First, the cost of charging will be reduced since the vehicle will charge in feasible hours when the electricity cost is lowest. Second, the device will decrease the risk of insufficient energy in the battery to drive; as opposed to a driver simply plugging in a PEV arbitrarily, the driver will be notified of the best times to plug-in to ensure the vehicle is sufficiently charged. The utilities will also benefit from a DR mechanism, since it will maintain the same peak power demand, whereas PEV charging without demand response will increase the peak power demand. Such a device is a practical extension of services that are currently provided with PEV charging stations, which allow the user to control charging but require settings to be determined by the user [16]. 
An aggregator's role is between these devices and the dispatcher to establish and monitor the market supply and vehicle demand. We assume such an aggregator will exist, and that it will be possible for users to communicate to the aggregator their expected driving schedule and vehicle characteristics. It should be mentioned that such managed charging is often envisioned to be an 'opt in' service, in which a consumer has the option to participate.

\subsection{Motivation}

It is projected that by the year 2030 , between $6 \%$ and $30 \%$ of vehicles in use will be PEVs [8]. These electric vehicles will each run on energy provided by a battery that will charge from the electricity grid, which suggests an increase in electricity demand. The Electric Power Research Institute (EPRI) showed in their work [8] that in the worst case, the increase in total grid resource capacity will be 5-6\%, and smart charging, i.e., shifting some charging to off-peak hours, will decrease the impact to only 1-2\%. In a collaborative work between Better Place and PJM Interconnection, Schneider et al. showed that such controlled charging will reduce consumer energy costs by $45 \%$ [21].

As more motivation, renewable energy sources are becoming increasingly prevalent. Many renewable power plants generate more electricity than is needed during off-peak hours, and a lack of sufficient energy storage means this surplus cannot always be stored for later use to meet the demand. For example, during the first half of 2008, an overproduction of wind power in West Texas led to negative electricity prices $20 \%$ of the time, and most of this surplus occurred in the middle of the night [24]. If vehicles had been connected to the grid with a DR mechanism, they may have actually profited from charging their batteries during these time periods.

\section{$1.2 \quad$ Related Work}

A number of papers in the field of electric transportation have established the benefits of smart charging, including $[4,8,17,25]$. However, there is currently no standard agreement on how to manage PEV charging. No previously proposed algorithm is based on a relatively small amount of information and dynamically creates charging schedules for thousands of vehicles in a fleet while meeting an external scheduling obligation.

Han et al. in [11] use dynamic programming to assign charging schedules that provide frequency regulation. Similarly, in the work by Wu et al. [26] an algorithm is constructed that makes dynamic decisions for lowest-cost charging schedules of PEVs. Neither of these works considers an obligation to the market that needs to be met, and the demand of each vehicle is considered individually (i.e., not as part of a fleet); both suggested algorithms would result in an increased peak demand. In [13], Ma et al. establish a decentralized algorithm that determines an equilibrium price so that the total amount of charging done in the fleet fills the 'overnight demand valley.' This algorithm takes into account all vehicles in the fleet, but is not dynamic (i.e., it is solves an offline problem) and assumes only a few types of driving behaviors exist. A dynamic (or online) algorithm does not require that all vehicles are connected to the grid at the same time to exactly report their future driving schedules, and makes decisions for each vehicle without solving a problem that depends on knowing the demands of the entire fleet.

Our algorithm depends only on historical data, or a few hundred driving behaviors from a previous similar day. The implementation is very simple: a set of unique adjusted 'prices' are generated for each driving behavior and charging is allocated among hours with the lowest adjusted

price value. This pricing scheme will distribute electricity to thousands of PEVs in a fleet while 
meeting an obligation to the market. It is assumed that vehicles will connect to the grid during periods when they are parked for at least an hour. We use real data on driving behaviors, electricity loads, electricity and gasoline pricing, and vehicle characteristics to generate our results.

\section{Problem Description}

An aggregator will make commitments to both the PEV consumers and their utility. To the utility, the aggregator will commit to ensuring the electricity supplied to the vehicles stays within a certain upper limit; we say that the market places a charge cap on the total amount of charging that can be done by the fleet in each hour, in order to stay within the given upper limit. In addition, vehicles will plug-in to the grid at various times and the aggregator will commit to providing each PEV with enough energy to drive. Thus, each vehicle's charging decision should:

1. be determined instantly as vehicles plug-in to the grid,

2. provide enough charge to each individual PEV to meet its daily transport load,

3. meet the obligation to the market,

4. consider the demand of the fleet as a whole, and

5. reduce the cost to the consumer.

Our algorithm is based on linear programming theory and achieves all of the above for a time period of $n$ hours in the future; the value of $n$ is easily adjustable. For instance, our results consider $n=120$ hours (or 5 days) in the future and solve the optimization problem (CLP) once at the start of the 5-day period to determine charging decisions for the next 120 hours. Vehicles are assumed to plug-in periodically over a given period of time (say, 12 hours) and report their driving schedule for the next $n$ hours. Moreover, we assume the information on the vehicle specifications can be obtained and that the aggregator has collaborated with the market to place a charge cap on the total amount of charging that can be done in each hour by the fleet. In the dynamic algorithm, each vehicle's charging schedule is instantly determined as it connects to the grid. We assume a few hundred (say, 400) driver behaviors from a similar day in a similar region are known, in addition to the predicted electricity load for the next $n$ hours.

\subsection{Linear Programming Formulation}

For explanation purposes, in the following linear programming formulation we assume perfect foresight so that the aggregator has all the information ahead of time (this is not assumed for our dynamic algorithm). Let $m$ be the number of vehicles and $n$ be the number of hours in the future considered. We write the driving load of each vehicle $j$ as a vector $t_{j} \in \mathbb{R}^{n}$, where the $h$ th element $t_{j h}$ is the number of miles driven in hour $h$. The aggregator also knows the electricity prices at each hour $p \in \mathbb{R}^{n}$, the gasoline prices $p^{g} \in \mathbb{R}^{n}$, the market's charge cap for each hour $c_{\text {cap }} \in \mathbb{R}^{n}$, and the characteristics of each vehicle. Our mechanism considers Plug-in Hybrid Electric Vehicles (PHEVs) that receive power from both gasoline and electricity, in addition to Battery Electric Vehicles (BEVs) that only receive power from electricity. A full list of parameters and variables is given in Table 1.

The following linear program (LP) will find feasible charging, fueling, and generating schedules, $c_{j}, f_{j}, g_{j} \in \mathbb{R}^{n}$, for each vehicle $j=1,2, \ldots, m$, such that each vehicle has enough energy in its battery to drive, while minimizing the cost and staying within the charge cap. It is assumed here 


\begin{tabular}{|c|l|c|l|c|}
\hline \multicolumn{2}{|c|}{ Variables } & \multicolumn{2}{|c|}{ Input Parameters } & Dimension \\
\hline$s_{j}$ & electricity storage & $\bar{s}_{j}$ & storage capacity & $n$ \\
\hline$c_{j}$ & charging schedule & $\bar{c}_{j}$ & maximum charging rate & $n$ \\
\hline$s_{j}^{g}$ & gasoline storage & $s^{g}{ }_{j}$ & gas tank capacity & $n$ \\
\hline$f_{j}$ & fueling schedule & $f_{j}$ & maximum fueling rate & $n$ \\
\hline$g_{j}$ & generation schedule & $\bar{g}_{j}$ & maximum generation rate & $n$ \\
\hline & & $t_{j}$ & driver transport load & $n$ \\
\hline & & $p, p^{g}$ & electricity and gasoline prices & $n$ \\
\hline & & $c_{\text {cap }}$ & charge cap & $n$ \\
\hline & & $s_{j 0}, s_{j 0}^{g}$ & initial battery and gasoline storage & 1 \\
\hline & & $c_{\text {eff }}, g_{\text {eff }}$ & charging and generating efficiencies & 1 \\
\hline
\end{tabular}

Table 1: List of Parameters and Variables

that the aggregator has perfect foresight for the $n$-hour period. We use MATLAB vector notation, where $x_{a: b}$ refers to the elements $\{a, a+1, \ldots, b\}$ of $x$.

$$
\begin{array}{cl}
\underset{c_{j}, f_{j}, g_{j}, \forall j}{\operatorname{minimize}} & \sum_{j=1}^{m}\left(p^{T} c_{j}+\left(p^{g}\right)^{T} f_{j}\right) \\
\text { subject to } & s_{j, 1: n}=s_{j, 0: n-1}+c_{\mathrm{eff}} \cdot c_{j}+g_{\mathrm{eff}} \cdot g_{j}-(\mathrm{kWh} / \mathrm{mile}) \cdot t_{j} \\
& s_{j, 1: n}^{g}=s_{j, 0: n-1}^{g}+f_{j}-(\mathrm{gallon} / \mathrm{kWh}) \cdot g_{j} \\
& t_{j h}=0 \Longrightarrow f_{j h}=0, g_{j h}=0 \\
& t_{j h}>0 \Longrightarrow c_{j h}=0 \\
& \sum_{j=1}^{m} c_{j} \leq c_{\mathrm{cap}} \\
& 0 \leq\left(s_{j}, c_{j}, s_{j}^{g}, f_{j}, g_{j}\right) \leq\left(\bar{s}_{j}, \bar{c}_{j}, \bar{s}_{j}^{g}, \bar{f}_{j}, \bar{g}_{j}\right)
\end{array}
$$

The first constraint updates the battery storage amount and the second updates the gasoline storage amount. The third and fourth constraints ensure that the vehicle can only fuel the tank and generate electricity when the vehicle is driving, and that the vehicle battery can only be charged when the vehicle is parked for at least an hour. The second to last constraint ensures that the total amount of charging done in each hour is below the charge cap, $c_{\text {cap }}$. And the last constraint ensures the variables stay within their physical bounds.

This problem formulation is static, since it assumes the aggregator knows the exact future driving schedules of all vehicles that plug-in to the grid. Moreover, our testing showed that solving this problem for a fleet of 10,000 vehicles over a 5-day period takes hours on a single workstation. Thus, instead of considering the micro-level linear program described above for all vehicles, we only consider a few hundred possible vehicle behaviors and their aggregated demands in this linear program. These data can be based on previous driving schedules from a relatively small population. After solving the aggregated linear program, we obtain adjusted 'prices' and use them to dynamically assign feasible and satisfactory charging schedules to thousands of individual vehicles within milliseconds of a connection to the grid.

\section{Clustering}

Our algorithm assigns charging schedules that consider possible driving behaviors of an entire fleet. Since it is impossible to predict the exact driving behaviors and available charging times 
of individual vehicles of a fleet, we use clustering to estimate the expected future demand. This approximation of future driving loads is necessary to stay below a given charge cap.

\subsection{Lowest-Cost Charging}

Allocating charge to each vehicle in the hours with the lowest electricity price will guarantee the lowest cost to each driver, but generally results in an increase in peak electricity demand. This is the method used in [26], and is not feasible when considering scheduling obligations. For example, consider the following scenario with two pure electric vehicles in a three hour period, where

$$
p=\left[\begin{array}{l}
0.10 \\
0.12 \\
0.14
\end{array}\right], \quad t_{1}=\left[\begin{array}{l}
0 \\
0 \\
1
\end{array}\right], \quad t_{2}=\left[\begin{array}{l}
0 \\
1 \\
0
\end{array}\right], \quad c_{\text {cap }}=(\mathrm{kWh} / \mathrm{mile}) \cdot\left[\begin{array}{l}
1 \\
1 \\
1
\end{array}\right], \quad \text { and } \quad s_{10}=s_{20}=0 .
$$

Assume both vehicles connect to the grid before hour 1, but at slightly different times, and charging schedules are allocated on a first-come, first-served basis. If vehicle 1 arrives first, an assignment based on electricity price will charge vehicle 1 in hour 1 , and the given charge cap implies there will be no more electricity available in hour 1 . When vehicle 2 plugs in slightly later, it will not be able to charge during hour 1 since the charge cap is already reached. Thus, vehicle 2 will not have sufficient battery energy storage to drive, and the algorithm has failed by not considering market constraints and the demand of future vehicles to connect. Note that because we assume a model where vehicles pay a low-cost flat rate for facilitated charging, vehicle 1 will not end up paying more than vehicle 2 and vice versa.

Note that for simplicity in explanation, we say in this example that the charge cap is in terms of $\mathrm{kWh}$ (energy), not $\mathrm{kW}$ (power), however, this is equivalent to saying the charge cap is a constant $1 \mathrm{~kW}$ of power, which totals to $1 \mathrm{kWh}$ of energy over each hour.

\subsection{Clustering Driving Patterns}

In order to consider future demands of vehicles in a fleet, a clustering algorithm is used to determine a number of different base driving profiles. We define a base driving profile to be the "best-fit" to a group of drivers with similar driving patterns. Each vehicle is then matched with the most similar base driving profile and properties of the corresponding cluster are used to determine the individual charging schedule.

Considering the approximate needs of each cluster before assigning individual schedules will ensure the demands of each vehicle can be met. Moreover, the aggregator can use the base driving profiles and the expected number of drivers in each cluster to approximate the energy demand of the fleet, which is useful in negotiations with the market on placing a charge cap (i.e., in determining scheduling obligations).

Real driving behavior data was used for the clustering and was obtained from the National Household Transportation Survey (NHTS) [22]. The survey contains the driving schedule of over 150,000 individuals, each for a 24 -hour period. We took data for each individual and put it into vector format, so each individual vehicle $j=1, \ldots, m$ has a vector of the form:

$$
t_{j}=\left[t_{j 1}, t_{j 2}, \cdots, t_{j, 24}\right] \in \mathbb{R}^{24} .
$$

For simulation purposes, these transport loads were assigned to either a PHEV or a BEV: if the daily transport load amounted to less than 70 miles, the corresponding vehicle was assumed to be a 
$\mathrm{BEV}$; otherwise, it was assumed to be a PHEV. Realistically, the vehicle type would be information passed from the drivers to the aggregator.

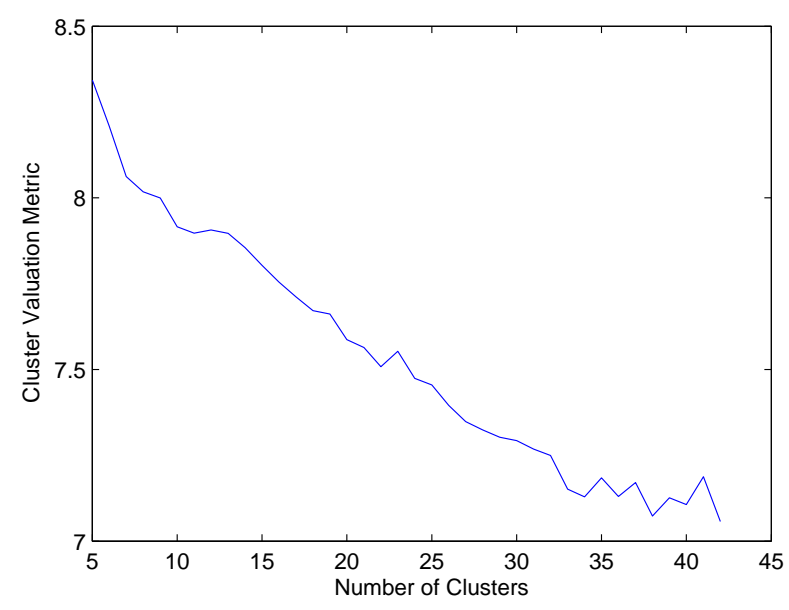

Figure 1: Cluster Valuation Metric for Given Numbers of Clusters Using $k$-means

The $k$-means clustering algorithm [12] with Euclidean distances is used on the transport load vectors. BEVs and PHEVs were separated before the clustering so that vehicles corresponding to the same base driving profile are either all BEVs or all PHEVs. Figure 1 shows the cluster valuation metric we used to determine the benefit of the number of clusters. Each cluster has a centroid (or mean), and we measure the distance from each point in the cluster to its centroid, called the withincluster mean. We then find the mean of these distances among all clusters, say the overall-cluster mean. Since $k$-means clustering is a randomized algorithm, we perform 50 runs of the algorithm on different fleets of transport loads $t_{j}$, and take the statistical mean of the overall-cluster mean over all the runs; the logarithm of this value is our cluster valuation metric on the $y$-axis of Figure 1 , for the range of 5 to 45 clusters. As Figure 1 shows, the valuation metric within-cluster means stop decreasing at $k=37$ and no more than 43 clusters can be formed; thus, we form 37 base driving profiles. By clustering 400 driving profiles from a similar day (i.e., same day of the week in the same region) into 37 base driving profiles and determining the portion of vehicles in each cluster, expected hourly demand of a fleet of 10,000 PEVs can be estimated.

\subsection{Dual Problem of the Clustered Formulation}

We form 37 clusters of driving behaviors for a similar previous $n$-hour period, and assign each cluster $\ell=1, \ldots, 37$ a weight $b_{\ell}$. This weight takes into account both the number of vehicles and the battery sizes of vehicles in the group, so that the demand of each cluster is proportionally accounted for. Since the base driving schedule of each cluster is known for the entire $n$-hour period, we can use them to form a static formulation as a linear program to determine feasible charging, fueling and generating schedules, $c_{\ell}, f_{\ell}, g_{\ell} \in \mathbb{R}^{n}$, while minimizing the cost and staying within the 
charge cap. This formulation will act as a proxy for the aggregate behavior of the actual fleet:

$$
\begin{array}{cll}
\underset{c_{\ell}, f_{\ell}, g_{\ell}, \forall \ell}{\operatorname{minimize}} & \sum_{\ell=1}^{k} b_{\ell} \cdot\left(p^{T} c_{\ell}+\left(p^{g}\right)^{T} f_{\ell}\right) & \\
\text { subject to } & s_{\ell, 1: n}=s_{\ell, 1: n-1}+c_{\mathrm{eff}} \cdot c_{\ell}+g_{\mathrm{eff}} \cdot g_{\ell}-(\mathrm{kWh} / \mathrm{mile}) \cdot \widehat{t_{\ell}} & : \lambda_{\ell}^{s} \\
& s_{\ell, 1: n}^{g}=s_{\ell, 1: n-1}^{g}+f_{\ell}-(\mathrm{gallon} / \mathrm{kWh}) \cdot g_{\ell} & : \lambda_{\ell}^{s} \\
& t_{\ell h}=0 \Longrightarrow f_{\ell h}=0, g_{\ell h}=0 & : \eta_{\ell}^{1}, \eta_{\ell}^{2} \\
& t_{\ell h}>0 \Longrightarrow c_{\ell h}=0 & : \eta_{\ell}^{3} \\
& \sum_{\ell=1}^{m} b_{\ell} \cdot c_{\ell} \leq c_{\mathrm{cap}} & : \theta \\
& 0 \leq\left(s_{\ell}, c_{\ell}, s_{\ell}^{g}, f_{\ell}, g_{\ell}\right) \leq\left(\bar{s}_{\ell}, \bar{c}_{\ell}, \bar{s}_{\ell}^{g}, \bar{f}_{\ell}, \bar{g}_{\ell}\right) & : \nu_{\ell}, \gamma_{\ell}
\end{array}
$$

where $\widehat{t_{\ell}} \in \mathbb{R}^{n}$ is the mean driving load of the vehicles in cluster $\ell$, for $\ell=1, \ldots, k$. The variables on the far right in (CLP) are the dual variables associated with each constraint, where

$$
\lambda_{\ell}^{s}, \lambda_{\ell}^{s^{g}} \in \mathbb{R}^{n+1}, \quad \eta_{\ell}^{1}, \eta_{\ell}^{2}, \eta_{\ell}^{3} \in \mathbb{R}^{n}, \quad \theta \in \mathbb{R}^{n},
$$

and

$$
\nu_{\ell}=\left(\nu_{\ell}^{s}, \nu_{\ell}^{c}, \nu_{\ell}^{s^{g}}, \nu_{\ell}^{f}, \nu_{\ell}^{g}\right) \in \mathbb{R}^{5 n+2}, \quad \gamma_{\ell}=\left(\gamma_{\ell}^{s}, \gamma_{\ell}^{c}, \gamma_{\ell}^{s^{g}}, \gamma_{\ell}^{f}, \gamma_{\ell}^{g}\right) \in \mathbb{R}^{5 n+2} .
$$

For simplicity, define the vector of all variables for each cluster as $x_{\ell}=\left(s_{\ell}, c_{\ell}, s_{\ell}^{g}, f_{\ell}, g_{\ell}\right) \in \mathbb{R}^{5 n+2}$. The linear program (CLP) has the dual problem:

$$
\begin{array}{lll}
\text { maximize } & \sum_{\ell=1}^{k}\left(\left(\lambda_{\ell}^{s}\right)^{T}\left[\begin{array}{c}
-s_{0 \ell} \\
\widehat{t_{\ell}}
\end{array}\right]+\left(\lambda_{\ell}^{s^{g}}\right)^{T}\left[\begin{array}{c}
-s_{0 \ell}^{g} \\
0
\end{array}\right]+\gamma_{\ell}^{T} x_{\ell}\right)+\theta^{T} c_{\mathrm{cap}} & \\
\text { subject to } & \lambda_{0: n-1, \ell}^{s}-\lambda_{1: n, \ell}^{s}+\gamma_{\ell}^{s}+\nu_{\ell}^{s}=0 & : s_{\ell} \\
& p-c_{\mathrm{eff}}^{\ell} \lambda_{1: n, \ell}-I_{d}^{\ell} \cdot \eta_{\ell}^{1}-b_{\ell} \cdot \theta+\gamma_{\ell}^{c}-\nu_{\ell}^{c}=0 & : c_{\ell} \\
& \lambda_{0: n-1}^{s^{g}}-\lambda_{1: n}^{s^{g}}+\gamma^{s^{g}}-\nu^{s^{g}}=0 & : f_{\ell} \\
& p_{g}-\lambda_{1: n, \ell}^{s^{g}}-I_{n d}^{\ell} \eta_{\ell}^{2}+\gamma_{\ell}^{f}-\nu_{\ell}^{f}=0 & : g_{\ell} \\
& -g_{\mathrm{eff}}^{\ell} \cdot \lambda_{1: n, \ell}^{s}-(\text { gallon } / \mathrm{kWh}) \cdot \lambda_{1: n, \ell}^{s^{g}}-I_{n d}^{\ell} \eta_{\ell}^{3}+\gamma_{\ell}^{g}-\nu_{\ell}^{g}=0 & : g_{\ell} \\
& \nu_{\ell}, \gamma_{\ell} \geq 0
\end{array}
$$

where $I_{d}^{\ell} \in \mathbb{R}^{n \times n}$ is the identity matrix with the columns corresponding to the hours cluster $\ell$ is not driving 'zeroed out', and $I_{n d}^{\ell} \in \mathbb{R}^{n \times n}$ is the identity matrix with the columns corresponding to hours the cluster is driving 'zeroed out', i.e., $I_{d}^{\ell}+I_{n d}^{\ell}=I_{n}$.

In contrast to the large problem (LP) with tens of thousands of vehicles, this formulation is considerably smaller, with one schedule per cluster. In our testing, once the number of clusters to form was determined, forming a set of clusters took around 0.3 seconds and solving the clustered linear program (CLP) to find the corresponding dual variables can be done in about 40 seconds on a single workstation. Moreover, the solution and dual variables are only found once, before the dynamic portion of the algorithm assigns charging schedules.

\section{Constraint-Adjusted Pricing}

Our dynamic algorithm uses properties of the dual problem (DCLP) to create adjusted 'prices' for each behavior cluster, so that allocating charging among the hours with the lowest adjusted price value will result in a feasible and satisfactory solution. These new prices are called constraintadjusted prices, since they use the dual variables in the constraints of (DCLP). Specifically, we use the second constraint in (DCLP) corresponding to the primal variable $c_{\ell}$,

$$
p-c_{\mathrm{eff}}^{\ell} \lambda_{1: n, \ell}-I_{d}^{\ell} \cdot \eta_{\ell}^{1}-b_{\ell} \cdot \theta+\gamma_{\ell}^{c}-\nu_{\ell}^{c}=0,
$$


where $\gamma^{c}, \nu^{c}$ are slack variables. By strict complementarity, for each hour $h=1, \ldots, n$,

$$
\begin{aligned}
\text { if }\left(p_{h}-c_{\mathrm{eff}}^{\ell} \cdot \lambda_{h \ell}-I_{d} \cdot \eta_{h \ell}^{1}-b_{\ell} \cdot \theta_{h}\right)>0, \text { then } c_{\ell h} & =0 \\
\text { and if }\left(p_{h}-c_{\mathrm{eff}}^{\ell} \cdot \lambda_{h \ell}-I_{d} \cdot \eta_{h \ell}^{1}-b_{\ell} \cdot \theta_{h}\right)<0, \text { then } c_{\ell h} & =\bar{c}_{\ell}
\end{aligned}
$$

where $\bar{c}_{\ell}$ is the maximum charging rate of cluster $\ell$. The value

$$
d_{\ell}:=p_{h}-c_{\mathrm{eff}}^{\ell} \cdot \lambda_{h \ell}-I_{d} \cdot \eta_{h \ell}^{1}-b_{\ell} \cdot \theta_{h}
$$

can be thought of as a vector of 'constraint-adjusted prices' over the next $n$ hours for cluster $\ell$ that takes into account: the charge cap, current electricity price, current battery energy storage, and the vehicle's driving schedule.

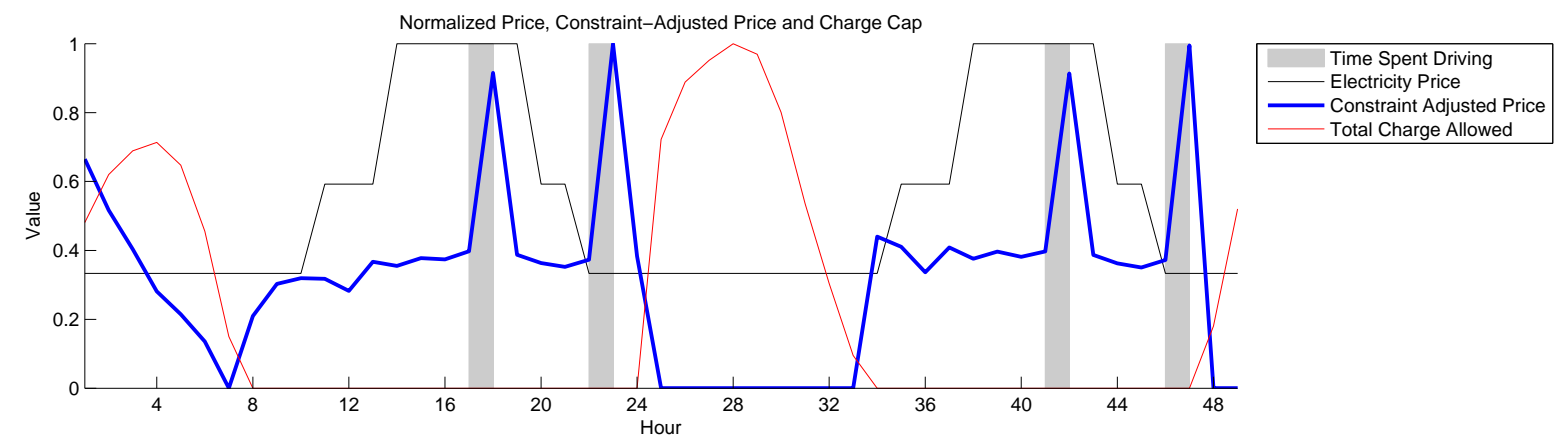

Figure 2: Comparison of the Normalized Constraint-Adjusted Price vs. Electricity Price

Figure 2 shows an example of the constraint-adjusted prices, along with the electricity price, total charge allowed, and driving schedule for a vehicle over a 2-day (or 48-hour) period, where each set of values is normalized by its respective largest element (i.e., normalized by the $\ell_{1}$-norm). Here, the 'total charge allowed' is the amount of electricity the scheduling obligation allows for the fleet to charge, i.e., it is the electricity load without PEVs subtracted from the charge cap. For this example, the driver's profile is assumed to be the same on each day. The electricity load is based on a week in August 2011 in the PG\&E transmission access charge area, and the prices used are PG\&E baseline summer time-of-use (TOU) rates. The charge cap at each hour is assumed to be the daily peak demand value; in broad terms, this will mean that the total charge allowed increases as the demand decreases, and no charging is allowed during the hour when total electricity demand reaches its peak.

Note that the constraint-adjusted price does not actually become zero; since these are the normalized values, this plot shows that the constraint-adjusted price becomes relatively very small when the supply is large. Furthermore, the constraint-adjusted price is relatively large during the first hours, because we assume each vehicle starts with a full battery.

The constraint-adjusted prices represent the cost to the aggregator of a vehicle charging in a certain hour when considering the above factors, as is shown in Figure 2. The price increases when the vehicle is driving, and when the charge cap is very small. Note that the constraint-adjusted price still takes into account the hourly electricity price, so the vehicle will first charge in the hours 
with the lowest cost, but the additional factors are also taken into account. A mechanism using the constraint-adjusted prices to determine vehicle charging, as opposed to electricity price, would assign charging schedules that satisfy both the consumers and the market.

As each vehicle plugs in to the grid, it reports its expected driving schedule for the next $n$ hours. Algorithm 1 uses these constraint-adjusted prices for the next $n$ hours to instantly determine lowcost feasible charging schedules. The mechanism will charge the battery (and notify the driver to fill the tank, if necessary) the amount needed to complete a given transport load, during the feasible hours where the constraint-adjusted price is lowest. Since Step 2 is executed only once and prices are constant over the horizon, the entire charging schedule for each vehicle is determined at the time it connects. This is based on an assumption that electricity prices are constant, which may be relaxed in future work.

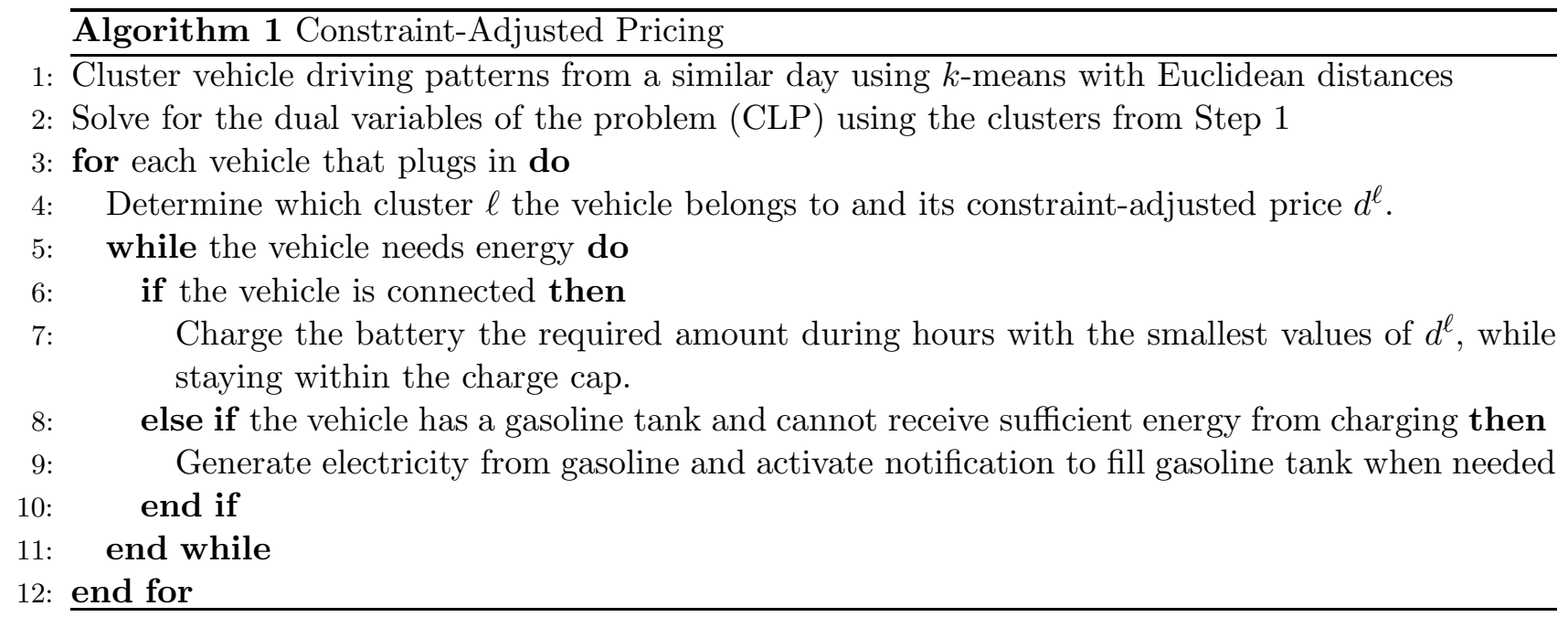

Revisiting the previous example of two vehicles in three hours, the constraint-adjusted prices for vehicle $j=1,2$ are

$$
d_{1}=\left[\begin{array}{c}
0.38 \\
0 \\
0.76
\end{array}\right], \quad d_{2}=\left[\begin{array}{c}
0 \\
0.76 \\
0.07
\end{array}\right]
$$

If the two vehicles are assigned charging schedules with constraint-adjusted prices via Algorithm 1 , vehicle 1 will charge in hour 2 (not hour 1, as before), allowing vehicles 2 to charge in hour 1.

\section{Comparison Algorithms}

We compare Constraint-Adjusted Pricing, Algorithm 1, to a number of other possible charging scenarios, resulting from either standard charging without a DR mechanism or from a DR mechanism that allocates charging differently. We compare three standard measurement and benefit features [10] from each charging scenario: cost to the consumer, increase in peak electricity demand and the total amount of energy used.

In general, the baseline for comparison is the estimate of electricity usage in the absence of a DR mechanism [7]. Since we are simulating a fleet of vehicles, we can compare the results of our algorithm to the outcome if vehicles were to charge each time they plug in for at least an hour 
(Algorithm 2, Standard Charging). We also compare our results to two potential DR mechanisms (Algorithm 3, Lowest-Cost Charging and Algorithm 4, Relative Primal Percentages). We briefly describe each algorithm used for comparison below before summarizing the results.

\subsection{Standard Charging}

For a standard baseline, we simulate the outcome of vehicles automatically charging each time they plug-in, until either the battery is full or the connection is ended; we assume that a vehicle is plugged in to the grid each time it is parked for at least an hour. This is called Standard Charging, as described in Algorithm 2, since these charging schedules are the outcome of an absence of DR intervention.

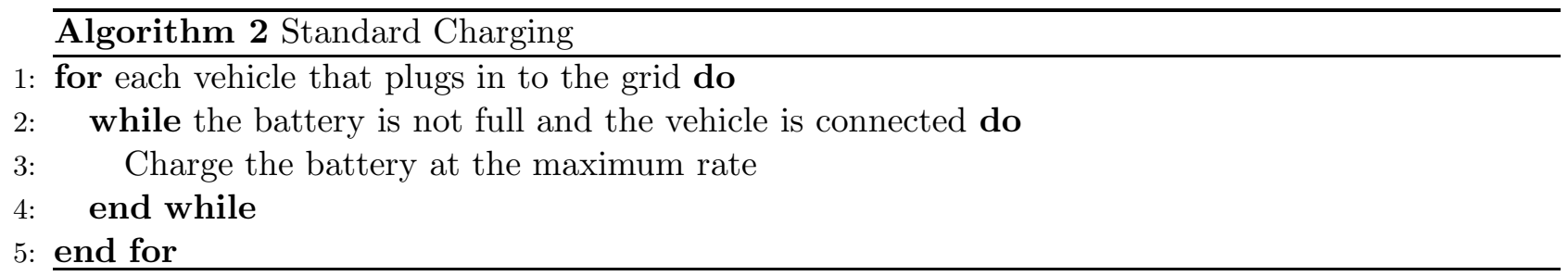

\subsection{Lowest-Cost Charging}

For a comparison to DR mechanisms that are currently in place, we simulate the algorithm where vehicles only charge when needed, in the hours with the lowest possible electricity price, as in Algorithm 3. This is equivalent to a user charging only in hours with the lowest electricity price (i.e., when the price is below a given threshold) unless such charging is not sufficient, in which case the battery is also charged in hours with a higher electricity price (i.e., when the price is above a given threshold). As described in [6], this is an Economic-based DR program, which is a concept used in practice with DR resources for the Commercial and Industrial sector.

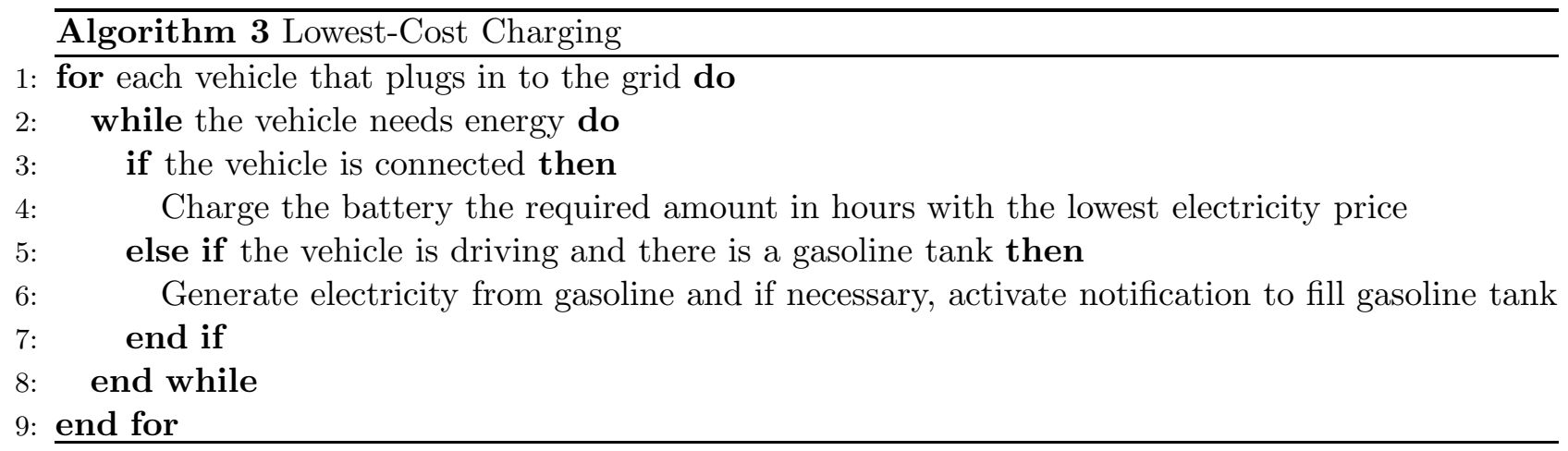

\subsection{Relative Primal Percentages}

The last algorithm we use for comparison uses the solution of the primal problem (CLP) to determine charging, generating and fueling schedules of a fleet of PEVs. The solution to the primal problem (CLP) takes into account the charge cap, electricity price, state of the battery, and driving 
schedule. Thus, we show that generating charging schedules using the primal solution as a guideline is not as effective as using information from the dual problem to construct Constraint-Adjusted Pricing.

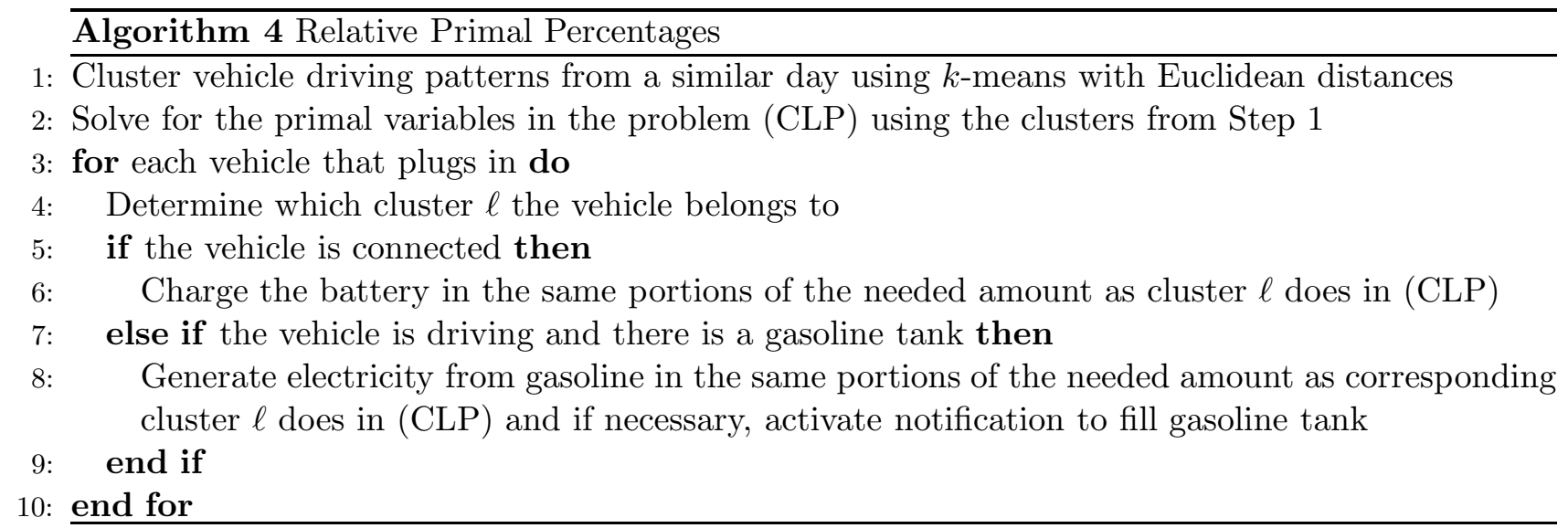

This algorithm assigns schedules so that each vehicle depends on power from charging, generating, and fueling in the same ratios and in the same relative percentage in each hour as in the primal. For example, if a cluster obtains $\alpha_{c} \%$ of its energy from charging, $\alpha_{g} \%$ from generating, and $\alpha_{f} \%$ from fueling, then so will every vehicle in this cluster. Moreover, if $r_{\ell 1}^{c}, r_{\ell 2}^{c}, \ldots r_{\ell n}^{c}$ are the respective ratios of total charging cluster $\ell$ does in each hour, where $\sum_{h=1}^{n} r_{\ell h}^{c}=1$, then the amount of charging in each hour for any vehicle in the cluster will be in the same ratios, within feasible bounds. Similarly, the vehicle will generate and fuel in the ratios $r_{\ell}^{g}, r_{\ell}^{f} \in \mathbb{R}^{n}$. Algorithm 4 is a DR algorithm based on the primal solution of (CLP) to determine what portion of the over all charging should be done in each hour.

\section{Results}

The following examples show the resulting charging schedules for a fleet of 10,000 PEVs over a 5-day period. We compare Algorithm 1 to the algorithms in Section 5 to determine the advantages of Constraint-Adjusted Pricing. We use real data, including:

- driving patterns in urban California, obtained from the NHTS dataset [22],

- electricity loads in the PG\&E transmission area for the week of August 22-28, 2011 [3],

- PG\&E baseline summer time-of-use rates (electricity pricing) [18],

- and the mean gasoline price in the zip code 94305 on August 31, 2011 [19].

Figures 3 and 4 are examples that show the charging demands of a fleet of 10,000 PEVs added to the electricity load, using each algorithm. Lowest-Cost Charging and Standard Charging clearly create new peak demands, while charging the fleet with Constraint-Adjusted Pricing ensures the load stays within the charge cap.

The statistics in Tables 2 and 3 are the mean values taken over 250 simulations of a fleet with 10,000 vehicles for 5 days, or 120 hours. Each simulation uses a different fleet of vehicle driving behaviors, sampled from the NHTS data. Note that the clusters are formed from a separate subset of the data that is not used for testing. We assume that there is one PEV for every 3 households (the 


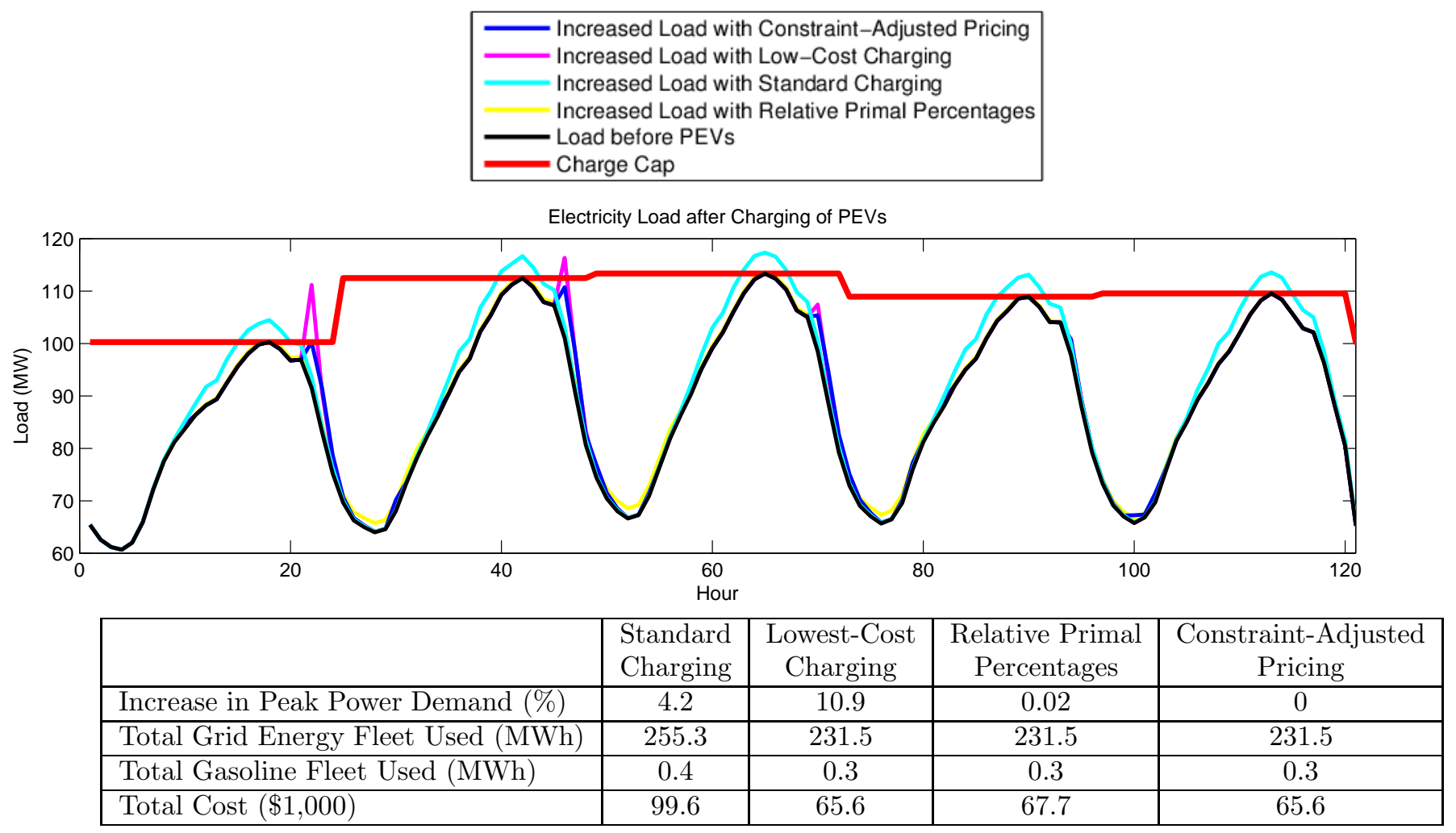

Figure 3: Charge cap at $100 \%$ of the peak

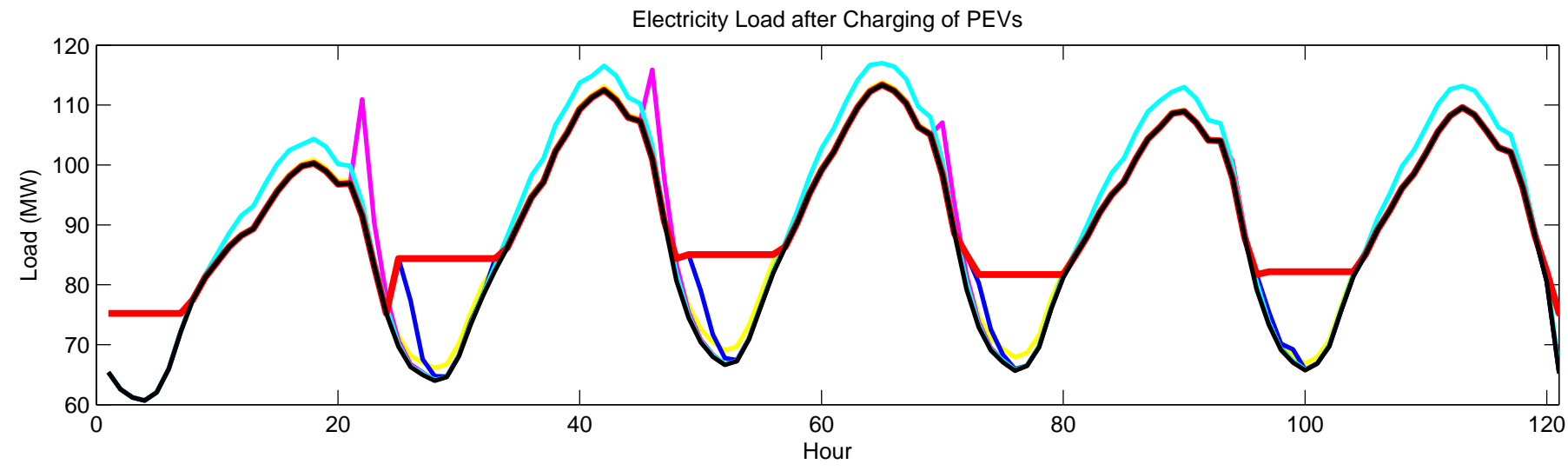

\begin{tabular}{|l|c|c|c|c|}
\hline & $\begin{array}{c}\text { Standard } \\
\text { Charging }\end{array}$ & $\begin{array}{c}\text { Lowest-Cost } \\
\text { Charging }\end{array}$ & $\begin{array}{c}\text { Relative Primal } \\
\text { Percentages }\end{array}$ & $\begin{array}{c}\text { Constraint-Adjusted } \\
\text { Pricing }\end{array}$ \\
\hline Increase in Peak Power Demand (\%) & 4 & 10.6 & 0.5 & 0 \\
\hline Total Grid Energy Fleet Used (MWh) & 254.2 & 230.7 & 230.9 & 230.7 \\
\hline Total Gasoline Fleet Used (MWh) & 0.4 & 0.3 & 0.3 & 0.3 \\
\hline Total Cost $(\$ 1,000)$ & 97.6 & 64.0 & 66.4 & 64.0 \\
\hline
\end{tabular}

Figure 4: Charge cap at $75 \%$ of the peak 
electricity demand is for 30,000 households) and that each vehicle plugs in over a 12-hour period, giving its expected driving schedule until the end of the fifth day. We also assume each vehicle has a full gasoline tank and battery at the beginning of the 5-day period. The costs listed below account for the cost of electricity and gasoline used to drive the vehicles, i.e. the costs account for new charging done and previously stored energy that is used for driving.

Constraint-Adjusted Pricing is the only mechanism simulated that does not create a new peak electricity demand. With a penetration of $33 \%$ of households owning a PEV, Standard Charging results in a 3.5\% increase in peak power demand, and Lowest-Cost Charging (of Economic-based DR programs) result in a $9.8 \%$ increase.

The total consumer cost of charging a PEV using Constraint-Adjusted Pricing is a reduction of more than $30 \%$ of the consumer cost from Standard Charging, and equal to the Lowest-Cost charging. However, Constraint-Adjusted Pricing is significantly better than Lowest-Cost Charging in terms of peak power increase, that is, Lowest-Cost Charging will not satisfy a market charge cap. Moreover, our aggregated linear program (CLP), which finds the constraint-adjusted prices, was solved in under a minute on a single workstation. Therefore, when considering consumer costs along with an increase in peak power demand, Constraint-Adjusted Pricing out-performs each algorithm in this comparison.

\begin{tabular}{|l|c|c|c|c|}
\hline & $\begin{array}{c}\text { Standard } \\
\text { Charging }\end{array}$ & $\begin{array}{c}\text { Lowest-Cost } \\
\text { Charging }\end{array}$ & $\begin{array}{c}\text { Relative Primal } \\
\text { Percentages }\end{array}$ & $\begin{array}{c}\text { Constraint-Adjusted } \\
\text { Pricing }\end{array}$ \\
\hline Increase in Peak Power Demand (MW) & 3.9 & 9.8 & $1.9 \mathrm{e}-2$ & 0 \\
\hline Percent Increase in Peak Power Demand (\%) & 3.5 & 9.8 & $1.7 \mathrm{e}-2$ & 0 \\
\hline Total Grid Energy Fleet Used (MWh) & 248.5 & 225.6 & 228.6 & 225.6 \\
\hline Total Gasoline Fleet Used (MWh) & 0.36 & 0.34 & 0.33 & 0.34 \\
\hline
\end{tabular}

Table 2: Power and Energy Comparisons: Mean Values

\begin{tabular}{|l|c|c|c|c|}
\hline & $\begin{array}{c}\text { Standard } \\
\text { Charging }\end{array}$ & $\begin{array}{c}\text { Lowest-Cost } \\
\text { Charging }\end{array}$ & $\begin{array}{c}\text { Relative Primal } \\
\text { Percentages }\end{array}$ & $\begin{array}{c}\text { Constraint-Adjusted } \\
\text { Pricing }\end{array}$ \\
\hline Total Cost $(\$ 1,000)$ & 98.5 & 64.9 & 65.7 & 64.9 \\
\hline Gasoline Cost $(\$ 1,000)$ & 47.7 & 44.6 & 43.7 & 44.6 \\
\hline Electricity Cost $(\$ 1,000)$ & 50.9 & 20.3 & 22.0 & 20.3 \\
\hline Mean Cost/Mile $(\$)$ & 0.069 & 0.030 & 0.031 & 0.030 \\
\hline
\end{tabular}

Table 3: Consumer Cost Comparisons: Mean Values

\section{Conclusions}

We constructed a dynamic mechanism to charge a fleet of PEVs. The allocation of electricity can satisfy an upper limit obligation to the market and provide each vehicle with enough energy for its transport load. Our algorithm depends only on the knowledge of a few hundred driving behaviors from a previous similar day and instantly assigns charging schedules to vehicles as they plug-in to the grid, without depending on information about the current transportation needs and driving schedules of the other vehicles in the fleet. We use a simple adjusted-pricing scheme to allocate 
charging to feasible and satisfactory hours. Our results show that Constraint-Adjusted Pricing out-performs each comparison algorithm when considering consumer cost, increase in peak power demand and total energy used.

Our future work will include incorporating vehicle-to-grid (V2G) capabilities of the fleet and ensuring our mechanism is robust to unexpected events. We also plan to determine an equilibrium price for the aggregator to use in determining PEV charging schedules, which will not change drastically due to slight fluctuations in demand.

\section{References}

[1] S. Agrawal, Z. Wang, and Y. Ye. A dynamic near-optimal algorithm for online linear programming. 2009. http://arxiv.org/abs/0911.2974.

[2] A. Brooks, E. Lu, D. Reicher, C. Spirakis, and B. Weihl. Demand dispatch. 8:20-29, May-June 2010 .

[3] CAISO. California ISO Oasis, 2011. http://www.oasis.caiso.com.

[4] ISO/RTO Council and KEMA. Assessment of plug-in electric vehicle integration with ISO/RTO systems. March 2010.

[5] E. Delage and Y. Ye. Technical report: A study of stochastic linear programs with cost uncertainty: Solution methods, distribution robustness, and implications for fleet mix optimization. Technical report, 2009.

[6] EnerNOC. Demand response: A multi-purpose resource for utilities and grid operators. Technical report, 2009.

[7] EnerNOC. The demand response baseline. Technical report, 2011.

[8] EPRI. Impact of plug-in electric vehicle technology diffusion on electricity infrastructure: Preliminary analysis of capacity and economic impacts. 2008. 1016853.

[9] EPRI. The power to reduce $\mathrm{CO}_{2}$ emissions: The full portfolio: 2009 technical report. 2009. 1020389.

[10] Federal Energy Regulatory Commission. 2008 Assessment of Demand Response \& Advanced Metering. Technical report, December 2008.

[11] Se. Han, So. Han, and K. Sezaki. Development of an optimal vehicle-to-grid aggregator for frequency regulation. IEEE Transaction on Smart Grid, 1:65-72, June 2010.

[12] Stuart P. Lloyd. Least squares quantization in PCM. IEEE Transactions on Information Theory, 28(2):129-137, March 1982.

[13] Zhongjing Ma, Duncan Callaway, and Ian Hiskens. Decentralized charging control for large populations of plug-in electric vehicles: Application of the Nash certainty equivalence principle. 2010 IEEE International Conference on Control Applications, pages 191-195, September 2010.

[14] National Energy Standards Board. DSM-EE glossary working group. Technical report, 2009. 
[15] National Energy Standards Board. NAESB Files Wholesale Electricity Demand Response Standards at FERC; Comparable Effort Now Under Way on Retail Side. Technical report, April 2009.

[16] Blink Network. Smart charging solutions at home. http://www.blinknetwork.com/chargers-residential.html.

[17] K. Parks, P. Denholm, and T. Markel. Costs and emissions associated with plug-in hybrid electric vehicle charging in the Xcel Energy Colorado service territory. May 2007.

[18] PG\&E. Electric schedule E-6: Residential time-of-use service, 2011. http://www.pge.com/tariffs/.

[19] San Jose Gas Prices. Palo Alto gas prices, 2011. http://www.sanjosegasprices.com/.

[20] N. Taheri R. Entriken and Y. Ye. Working technical report: Disaggregation of supply to individual plug-in electric vehicles. Technical report, 2011.

[21] S. J. Schneider, R. Bearman, H. McDermott, X. Xu, S. Benner, and K. Huber. An assessment of the price impacts of electric vehicles on the PJM market. May 2011.

[22] Federal Highway Administration U.S. Department of Transportation. 2009 National Household Travel Survey, 2009.

[23] U.S. Energy Information Administration. 2009. http://www.eia.gov/electricity/data.cfm.

[24] Ucilia Wang. Texas wind farms paying people to take power. December 2008. http://www.greentechmedia.com/articles/read/texas-wind-farms-paying-people-to-take-power-5

[25] R. Watts. Effects of plug-in hybrid electric vehicles on Vermont electric transmission system. Transportation Research Board 88th Annual Meeting, 2009.

[26] D. Wu, D. C. Aliprantis, and L. Ying. Load scheduling and dispatch for aggregators of plug-in electric vehicles. IEEE Transactions on Smart Grid, Special Issue on Transportation Electrification and Vehicle-to-Grid Applications, May 2011. 